Article

\title{
Nonlinear Optical Study in a Set of Dibenzylideneacetone Derivatives with Potential for Optical Frequency Conversion
}

\author{
Francisco A. Santos ${ }^{1,2}$, Luis M. G. Abegão ${ }^{3, * \mathbb{C}}$, Ruben D. Fonseca ${ }^{4}$, Aline M. Alcântara ${ }^{5} \mathbb{D}$, \\ Cleber R. Mendonça ${ }^{6}{ }^{(D)}$, Márcio A. R. C. Alencar ${ }^{1,2}{ }^{(D)}$, Marcelo S. Valle ${ }^{5}$, Kenji Kamada ${ }^{7}$, \\ Leonardo De Boni ${ }^{6}(\mathbb{D})$ and José J. Rodrigues Jr. ${ }^{1,2}$ (D) \\ 1 Departamento de Física, Universidade Federal de Sergipe, São Cristovão SE 49100-000, Brazil; \\ assis2091@academico.ufs.br (F.A.S.); marca.ufs@gmail.com (M.A.R.C.A.); joatan@ufs.br (J.J.R.J.) \\ 2 Laboratório de Corrosão e Nanotecnologia, Núcleo de Petróleo e Gás, Universidade Federal de Sergipe, \\ São Cristovão SE 49100-000, Brazil \\ 3 Department of Radiology and Biomedical Imaging, School of Medicine, Yale University, 300 Cedar Street, \\ New Haven, CO 06520, USA \\ 4 Departamento de Fisica, Universidad Popular del Cesar, Barrio Sabana, \\ Valledupar 2000004, Cesar, Colombia; rubenzfonseca@gmail.com \\ 5 Departamento de Ciências Naturais, Universidade Federal de São João del Rei, \\ São João del Rei MG 36301-160, Brazil; alinealcantara97@hotmail.com (A.M.A.); \\ marcelovalle@gmail.com (M.S.V.) \\ 6 Instituto de Física de São Carlos, Universidade de São Paulo, CP 369, São Carlos SP 13560-970, Brazil; \\ crmendon@ifsc.usp.br (C.R.M.); deboni@ifsc.usp.br (L.D.B.) \\ 7 IFMRI, National Institute of Advanced Industrial Science and Technology, Ikeda, Osaka 563-8577, Japan; \\ k.kamada@aist.go.jp \\ * Correspondence: luis.abegao@yale.edu or lma@campus.fct.unl.pt
}

Received: 30 November 2019; Accepted: 6 January 2020; Published: 9 January 2020

\begin{abstract}
The search for advanced optical materials, in particular, materials with nonlinear optical responses, has, in the last years, experienced substantial growth due to their vast applications in the photonics field. One of those applications is ultra-fast optical frequency conversion, in the optics communications field. Organic compounds have emerged as promising candidates for raw materials to develop nonlinear optical devices, such as optical converters, due to their intrinsic ultra-fast electronic responses. Also, the easy tailoring of organic molecular structures makes organic materials much more appealing than the inorganic ones. In this work, we have performed a linear and nonlinear optical characterization of a set of dibenzylideneacetone derivatives. The nonlinear optical responses investigated correspond to second- and third-order nonlinear processes, namely, first electronic molecular hyperpolarizability and two-photon absorption cross-section, respectively. The value of the first electronic molecular hyperpolarizability, up to $52 \mathrm{~cm}^{4} \cdot$ statvolt ${ }^{-1}$, could be considered a robust value when compared to the short-sized $\pi$-electron backbone length of the studied compounds. Such results suggest that these compounds exhibit the potential to be used as optical frequency converters. Quantum chemical calculations were used to predict the theoretical value of the first molecular hyperpolarizability, as well as to simulate the one- and two-photon absorption spectra for all compounds.
\end{abstract}

Keywords: optical communications; frequency converters; organic compounds; dibenzylideneacetone derivatives; second harmonic generation; two-photon absorption; quantum chemical calculations 


\section{Introduction}

The evolution of optical communication networks demands the search and development of raw materials with particular types of nonlinear optical (NLO) properties with the potential to be used as optical communication devices. Frequency converters and optical limiters are two examples of NLO properties of interest to search for [1-5]. It is in this context that organic compounds have an important role as raw materials for developing photonic devices to be applied in optical communications. Such an important role is defined by their ease of synthesis, ultrafast-response time, low cost, and crystal growth [6,7]. Moreover, organic compounds can also be tailored to have a broad spectral region of transparency in the visible region, which is crucial for applications in optical communications, such as all-optical signal processing and all-optical switching [8].

One of the NLO effects responsible for frequency conversion is the second-harmonic generation (SHG) of the incident light. This effect can be quantified by the determination of the first-order hyperpolarizability of individual organic molecules. One way to optimize the SHG effect is to increase the electronic hyperpolarizability of the molecular structure, which can be achieved, for example, by increasing the $\pi$-conjugation length. It is legitimate to think that compounds with an extensive $\pi$-conjugation length would be the ideal raw compounds for potential frequency converters. However, larger molecules are generally more challenging to combine in a dense solid-state, such as a crystal [9-11], representing a drawback to achieving the final photonic product. Another approach for improving the performance of the SHG response is to modify the charge density asymmetry of the compound by adding donor and acceptor peripherals groups on its molecular structure [12-14]. This latter approach probably will not be as effective in terms of achieving high values of polarizability, as is the case with increasing the $\pi$-conjugation length, but will overcome the issue of growth crystal, allowing one to reach a final product with more ease.

These strategies also cause changes in second-order hyperpolarizability, responsible for third-order nonlinear optical effects, such as the absorption of two photons. One of the likely applications of two-photon absorption (TPA) is optical limiting, which can be used as power regulation and protection devices in optical networks [15-18].

Previous works, both experimental and theoretical, have shown that dibenzylideneacetone derivatives have second- and third-order nonlinear optical properties of promising magnitude for photonic applications, such as optical frequency converters, optical switching, and optical limiting [19-23]. These compounds stand out for their high synthetic versatility, which allows new structures to be obtained and can serve as channels for the optimization of nonlinear optical properties. Moreover, the low coefficient of molar absorptivity in the visible (VIS) and near-infrared region (NIR), coupled with the ease of crystallization in the noncentrosymmetric form, makes these types of compounds potential candidates for nonresonant photonic applications [24-26].

From this perspective, we report the second- and third-order nonlinear optical properties of the dibenzylideneacetone-substituted compounds in a dichloromethane solution. The first molecular hyperpolarizability ( $\beta$ ) was obtained by using the hyper-Rayleigh scattering (HRS) technique, with a fixed excitation wavelength at the NIR. The experimental TPA cross-section $\left(\sigma^{\mathrm{TPA}}\right)$ spectra were measured with femtosecond pulses in the VIS region by the Z-scan technique. To further understand the nonlinear optical properties of the compounds, quantum chemical calculations (QCC) were made by employing the time-dependent density functional theory (TD-DFT) to calculate the components of tensor first molecular hyperpolarizability, as well as the transitions frequencies.

\section{Materials and Methods}

\subsection{Compounds}

The molecular structures of the studied samples are shown in Figure 1. The synthesis procedure for every sample was the following: into a round-bottom flask, a solution of the aromatic aldehyde (2-methoxybenzaldehyde: $1.20 \mathrm{~mL}$; 4-methoxybenzaldehyde: $1.22 \mathrm{~mL}$; 4-fluorobenzaldehyde: 0.79 
$\mathrm{mL}, 10 \mathrm{mmol})$ in ethanol $(10 \mathrm{~mL})$ was added to a solution of acetone $(0.29 \mathrm{~g}, 5 \mathrm{mmol})$ in sodium hydroxide $(0.40 \mathrm{~g}, 10 \mathrm{mmol})$ in ethanol $(10 \mathrm{~mL})$ at $0{ }^{\circ} \mathrm{C}$ and allowed to come to room temperature. The reaction mixture was stirred between 15 to $60 \mathrm{~min}$ and monitored by thinlayer chromatography (TLC) to confirm the consumption of the starting material. The precipitate was filtered and/or a portion of cold mixture of ethanol/water added to force the recrystallization and to yield dibenzylideneacetone (DBA) derivatives (4-DMDBA: 91\% yield, $1.34 \mathrm{~g}$, $4.55 \mathrm{mmol}$; 2-DMDBA: 56\% yield, $0.82 \mathrm{~g}, 2.79 \mathrm{mmol}$; 4-DFDBA: $92 \%$ yield, $1.24 \mathrm{~g}$, $4.59 \mathrm{mmol}$, respectively). The samples were characterized by nuclear magnetic resonance (NMR) spectroscopies. The ${ }^{1} \mathrm{H}$ NMR (500 MHz) and ${ }^{13} \mathrm{C} \mathrm{NMR}(125 \mathrm{MHz})$ spectra were recorded as solutions in deuterated chloroform $(\mathrm{CDCl} 3)$ on a Bruker spectrometer. The chemical shifts were expressed as $\delta$ (in ppm) with respect to a standard internal tetramethylsilane (TMS) reference $\left({ }^{1} \mathrm{H}\right.$ NMR). The full data regarding NMR spectroscopy can be found in the Supplementary Material.<smiles>[R]c1ccc(/C=C/C(=O)/C=C/c2ccc([R])cc2[R])c([R])c1</smiles>

$\begin{array}{lcc} & \mathrm{R}_{1} & \mathrm{R}_{2} \\ \text { 4-DMDBA } & \mathrm{OMe} & \mathrm{H} \\ \text { 2-DMDBA } & \mathrm{H} & \mathrm{OMe} \\ \text { 4-DFDBA } & \mathrm{F} & \mathrm{H}\end{array}$

Figure 1. The molecular structure of the dibenzylideneacetone derivatives.

\subsection{Optical Measurements}

All samples were dissolved in dichloromethane in a concentration of about $10^{-5} \mathrm{~mol} \mathrm{~L}^{-1}$ and stored in 10-mm thick fused quartz cuvettes, which were used to perform the linear optical measurements, such as the absorbance and the fluorescence emissions. Regarding the nonlinear optical measurements, the same optical path cuvette was used to determine the $\beta_{\text {HRS }}$ of the DBA derivatives, but with different molar concentrations ranging from $10 \mu \mathrm{M}$ to $50 \mu \mathrm{M}$. A short optical path cuvette (2-mm) was used to achieve the TPA, with a molar concentration of $50 \mu \mathrm{M}$.

A UV-VIS spectrophotometer (Shimadzu UV-1800) was used to record the absorbance of the samples, i.e., their one-photon absorption (OPA). A fluorescence spectrophotometer (Hitachi F4500) was used to measure the fluorescence emissions of all samples, but no shreds of evidence of any emission signals were observed.

To obtain the $\beta_{\text {HRS }}$ of the DBA derivatives dissolved in dichloromethane, we used an extension of the HRS technique introduced by Franzen et al. [27]. The light source employed was a solid-state laser (Nd:YAG), Q-switched and mode-locked laser operating at $1064 \mathrm{~nm}$, with a repetition rate of $300 \mathrm{~Hz}$. The system provides a light beam shaped by a pulse-train with about 30 pulses, in which every single pulse has an full width at half maximum (FWHM) of $100 \mathrm{ps,} \mathrm{separated} \mathrm{by} \mathrm{approximately}$ 13 ns. To prevent the sample from being exposed for a long time to the laser light, we used a computer-controlled shutter to block the laser beam during the dark count rate evaluation, as well as after the HRS measurements. Two crossed polarizers controlled the light beam pumping intensity, while a fast photomultiplier tube (PMT) placed perpendicularly to the pumping beam direction acquired the HRS signal. To improve the signal-to-noise ratio, a spherical mirror was used to collect the backscattering HRS light and direct it to the PMT. A $532 \mathrm{~nm}$ narrow bandpass filter, with a $10 \mathrm{~nm}$ bandwidth, was used to make sure that only the SHG signal to be detected by the PMT. More details about the experimental setup can be found elsewhere [27-29].

Assuming that all molecules of a binary solution (solute-solvent) do not have any absorption at the fundamental pumping frequency $\left(\mathrm{I}_{\omega}\right)$, it is accepted that the magnitude of the scattered signal at double-frequency $\left(I_{2 \omega}\right)$ ia related to $I_{\omega}$ by Equation (1) [30]. G is a coefficient related to the scattering geometry, local field factors at $\omega$ and $2 \omega$, as well as other optical and experimental conditions. $\mathrm{N}$ corresponds to the fractional concentration of the used samples, while $\left\langle\beta_{H R S}^{2}\right\rangle$ is the squared value of the orientationally-averaged first molecular hyperpolarizability. The subscripts $\mathrm{s}$ and $\mathrm{m}$ refer to the solvent and molecule (solute) investigated, respectively. 


$$
I_{2 \omega}=G\left(N_{s}\left\langle\beta_{H R S}^{2}\right\rangle_{s}+N_{m}\left\langle\beta_{H R S}^{2}\right\rangle_{m}\right) I_{\omega}{ }^{2}
$$

Experimental parameters such as the polarization state of both fundamental and second-harmonic beams, the molecular symmetry, and the geometry of the experimental setup [31], are essential parameters needed to obtain the most reliable relation between the $\left\langle\beta_{H R S}^{2}\right\rangle$ and the molecular tensor components $\left\langle\beta_{i j k}\right\rangle$. In the HRS experiment conducted in this work, the signal on frequency-doubled light scattered was collected under an angle of $90^{\circ}$ to the pumping light beam. In this situation, we can assume that the incident light beam propagation is in the $\mathrm{X}$-direction, polarized in the Z-direction, and detected in Y-direction. The dependence between the $\beta_{H R S}$ and the orientationally macroscopic hyperpolarizabilities averaged are given by the Equation (2) [32]. The laboratory coordinate system of reference was defined by the coordinates $(X, Y, Z)$, while the molecular coordinate system of reference was defined by $(x, y, z)$. Assuming that the fundamental and second-harmonic frequencies are far from the resonance frequencies, then Kleinman's symmetry condition can be applied. In this situation, the macroscopic hyperpolarizabilities averages $\left\langle\beta_{Z Z Z}^{2}\right\rangle$ and $\left\langle\beta_{Z X X}^{2}\right\rangle$ can be determined by using Equations (2a) and (2b), respectively [33].

$$
\begin{gathered}
\left\langle\beta_{H R S}\right\rangle=\sqrt{\left\langle\beta_{\mathrm{ZZZ}}^{2}\right\rangle+\left\langle\beta_{\mathrm{XZZ}}^{2}\right\rangle} \\
\left\langle\beta_{\mathrm{ZZZ}}^{2}\right\rangle=\frac{1}{7} \sum_{\zeta}^{x, y, z} \beta_{\zeta \zeta \zeta}^{2}+\frac{6}{35} \sum_{\zeta \neq \eta}^{x, y, z} \beta_{\zeta \zeta \zeta \beta_{\zeta \eta \eta}}+\frac{9}{35} \sum_{\zeta \neq \eta}^{x, y, z} \beta_{\eta \zeta \zeta}^{2}+\frac{6}{35} \sum_{\zeta \neq \eta \neq \xi}^{x, y, z} \beta_{\zeta \zeta \eta} \beta_{\eta \xi \xi}+\frac{12}{35} \beta_{\zeta \eta \xi}^{2} \\
\left\langle\beta_{Z X X}^{2}\right\rangle=\frac{1}{35} \sum_{\zeta}^{x, y, z} \beta_{\zeta \zeta \zeta}^{2}-\frac{2}{105} \sum_{\zeta \neq \eta}^{x, y, z} \beta_{\zeta \zeta \zeta \beta_{\zeta \eta \eta}}+\frac{11}{105} \sum_{\zeta \neq \eta}^{x, y, z} \beta_{\zeta \zeta \eta}^{2}-\frac{2}{105} \sum_{\zeta \neq \eta \neq \xi}^{x, y, z} \beta_{\zeta \zeta \eta} \beta_{\eta \xi \xi}+\frac{8}{35} \beta_{\zeta \eta \xi}^{2}
\end{gathered}
$$

The experimental determination of the two-photon absorption spectra of the DBA derivatives dissolved in dichloromethane was achieved by using a wavelength-tunable femtosecond Z-scan technique on the open-aperture mode [34]. The samples were excited by $120 \mathrm{fs}$ pulses from an optical parametric amplifier (Quantronix, model TOPAS) pumped by a Ti:Sapphire pulse amplified laser system $(775 \mathrm{~nm}$ and $1 \mathrm{kHz}$ repetition rate), allowing wavelength tuning from 480 up to $790 \mathrm{~nm}$. A spatial filter was placed in the output of the optical parametric amplifier, but before sample excitation, provided a Gaussian beam spatial profile excitation light beam. A more detailed description related to the experimental setup can be found in reference [28].

Assuming that the excitation beam has a Gaussian temporal profile, the relation between the normalized transmittance and sample position measured through Z-scan technique is given by [34]:

$$
T(z)=\frac{1}{\sqrt{\pi} q_{0}(z, 0)} \int_{-\infty}^{+\infty} \ln \left[1+q_{0}(z, 0) e^{-\tau^{2}}\right] d \tau
$$

in which, $q_{0}(z, 0)=\alpha_{2} I_{0} L\left(1+z^{2} / z_{0}^{2}\right)^{-1}, \alpha_{2}$ is the TPA coefficient, $I_{0}$ is the intensity of the pulse, $L$ is the optical path, $z_{0}$ is the Rayleigh length, and $z$ is the sample position. The $\sigma^{T P A}$ values were obtained using $\sigma^{2 P A}=\hbar \omega \alpha_{2} / N$, in which $\hbar \omega$ is the energy of the incident photon and $N$ is the density of molecules. Usually, $\sigma^{T P A}$ values are expressed in Göppert-Mayer units (GM), in which $1 \mathrm{GM}=1 \times 10^{-50} \mathrm{~cm}^{4} \cdot \mathrm{s} \cdot$ photon $^{-1} \cdot \mathrm{molecule}^{-1}$. The experimental Z-scan signatures corresponding to the maximum of TPA band from both molecular structures are presented in Figures S7-S9 of the Supplementary Material.

\subsection{Theoretical}

The OPA and the TPA spectra, as well as the values of the $\beta_{H R S}$, were calculated by using quantum-chemical calculations (QCC) performed with the time-dependent density functional theory 
(TD-DFT) [35] for the OPA/TPA, while the DFT [36] was used for the calculation of the $\beta_{H R S}$ tensor components. Three families of methods, combined with one split-valence basis set, were used to simulate and predict the linear and nonlinear optical responses by using the Gaussian 16 program package [37]. The exchange-correlation functional Becke, three-parameter, and Lee-Yang-Parr (B3LYP) [38], as well as its long-range corrected version, which includes the Coulomb-attenuating method (CAM-B3LYP) [39], were used. Moreover, a variation of a hybrid functional by Truhlar and Zhao (M06-2X) [40], which optimized the prediction of non-covalent interactions, was also used. A split-valence basis set with one additional polarization function for non-hydrogen atoms, one additional polarization function on hydrogen atoms, and with diffuse functions on non-hydrogen atoms $(6-311++(2 d, p))$, was applied together with the families of methods described previously. Geometry optimization calculations were performed in dichloromethane medium employing a polarizable continuum model (PCM), using the integral Equation formalism variant (IEF-PCM) [41]. All optimized geometries are presented in the Supplementary Material (Tables S1 and S2).

The simulation of the static $(\omega=0$ a.u.) and dynamic $(\omega=0.04282$ a.u. $)$ components of the first-order hyperpolarizability tensor $\left(\beta_{i j k}\right)$ in solvent were achieved through the combination of the keywords POLAR and CPHF, by using the optimized geometries. For molecular structures with very low symmetry, the hyperpolarizability tensor could be estimated more accurately by using the mixed spherical-Cartesian formalism [42]. In this formalism, the orientational averaged first-order hyperpolarizability squared $\left\langle\beta_{H R S}\right\rangle$ is expressed by Equation (4), in which the molecular $\beta_{H R S}$ tensor (third rank tensor) is decomposed as the sum of dipolar $(J=1)$ and an octupolar $(J=3)$ tensorial components [33]. The relationships between the dipolar and octupolar components and the Cartesian components of $\beta$ are described in Equations (4a) and (4b). A combination of Equations (4), (4a) and (4b) was used to estimate the theoretical values for the static and dynamic first-order hyperpolarizabilities.

$$
\begin{gathered}
\beta^{H R S}=\sqrt{\frac{2}{9}\left|\beta_{J=1}\right|^{2}+\frac{2}{21}\left|\beta_{J=3}\right|^{2}} \\
\left|\beta_{J=1}\right|^{2}=\frac{3}{5} \sum_{i} \beta_{i i i}^{2}+\frac{6}{5} \sum_{i \neq j} \beta_{i i i} \beta_{i j j}+\frac{3}{5} \sum_{i \neq j} \beta_{i j j}^{2}+\frac{3}{5} \sum_{i \neq j \neq k} \beta_{i j j} \beta_{i k k} \\
\left|\beta_{J=3}\right|^{2}=\frac{2}{5} \sum_{i} \beta_{i i i}^{2}-\frac{6}{5} \sum_{i \neq j} \beta_{i i i} \beta_{i j j}+\frac{12}{5} \sum_{i \neq j} \beta_{i j j}^{2}-\frac{3}{5} \sum_{i \neq j \neq k} \beta_{i j j} \beta_{i k k}+\sum_{i \neq j \neq k} \beta_{i j k}^{2}
\end{gathered}
$$

Time-dependent (TD) calculations were performed to simulate the OPA/TPA by using the optimized geometries. Tamm-Dancoff approximation (TDA) [43] was used to calculate the transition dipole moments from the ground state to the excited states, transition dipole moments between excited states, and the permanent dipole moments in the ground state and excited states, in order to obtain the dipole moments difference and the excitation energies. The spectral shape of the simulated spectra was obtained by using the sum-over-states (SOS) method reported previously [44]. The lowest fifteen excited states used to simulate the OPA/TPA spectra are shown in the Supplementary Material (Tables S3-S6).

\section{Results and Discussion}

\subsection{First Molecular Hyperpolarizability}

The OPA spectra of all DBA derivatives in dichloromethane solvent medium presented a broad band in the UV region with a molar absorption coefficient $(\varepsilon)$ of about 2 to $2.5 \times 10^{4} \cdot \mathrm{M}^{-1} \cdot \mathrm{cm}^{-1}$, as can be seen in Figure 2. The $\varepsilon$ value is neglible for wavelengths longer than $450 \mathrm{~nm}$, making these samples extremely suitable for HRS measurement when pumped with a pulsed laser at $1064 \mathrm{~nm}$. Figure 3 shows the HRS signal $(\mathrm{I}(2 \omega))$ of the compound 4-DMDBA for five different concentrations as a function of the pumping intensity $(\mathrm{I}(\omega))$. In addition, the linear dependence was observed between the relation 
$\mathrm{I}(2 \omega) / \mathrm{I}^{2}(\omega)$ and the sample concentration, as can be seen in inset of Figure 3. In this work, we use the external reference method to obtain the experimental factor $G$, in which the reference compound chosen was the para-nitroaniline (PNA). The $\beta_{H R S}$ value of the PNA is approximately $17 \times 10^{-30} \mathrm{~cm}^{4} \mathrm{statvolt}^{-1}$ in dichloromethane [45]. From the experimental results, the first molecular hyperpolarizability can be obtained through Equation (5), which relates the angular coefficient $(\alpha)$ of the samples with the one obtained from reference compound. The linear fitting presented in the inset of Figure 3 retrieves the $\alpha$ values of all compounds.

$$
\beta_{\text {sample }}=\beta_{\text {reference }} \sqrt{\frac{\alpha_{\text {sample }}}{\alpha_{\text {reference }}}}
$$

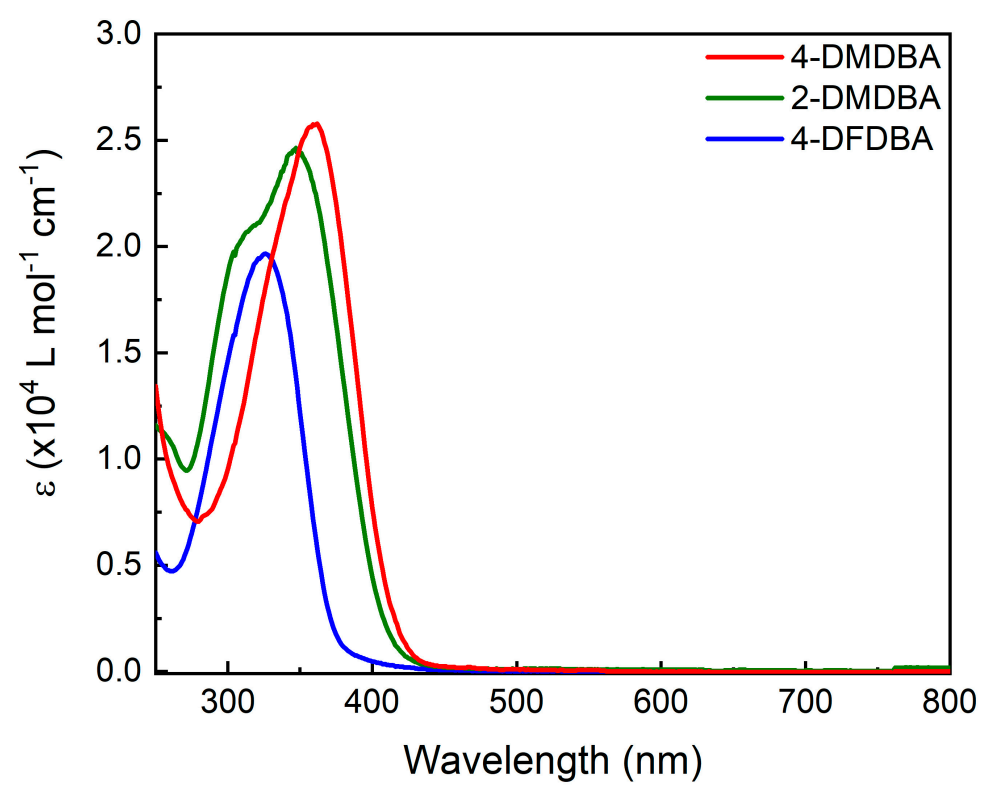

Figure 2. The molar absorption coefficient $(\varepsilon)$ as a function of the wavelength of all studied compounds.

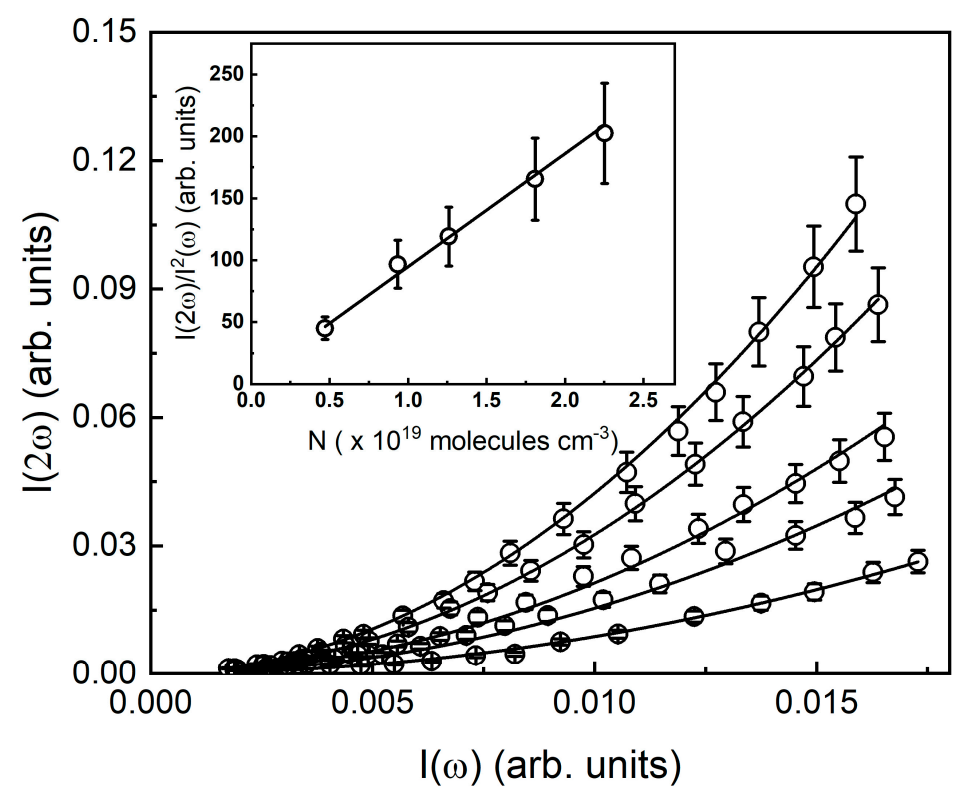

Figure 3. HRS signal as quadratic function of fundamental light beam irradiance for 4-DMDBA dissolved in dichloromethane (open circles). The inset shows the linear dependence between the $\left(\mathrm{I}(2 \omega) / \mathrm{I}^{2}(\omega)\right.$ and the density of molecules. 
Due to the same $\pi$-conjugated core-chain length of the studied samples, i.e., dibenzylideneacetone, the majority changes in the $\beta_{H R S}$ value are associated with the action and position of its peripherals groups. The highest $\beta_{H R S}$ value obtained was the 4-DMDBA compound, which is three times higher than the reference compound, justified by the para-position of the methoxy group. Compound 2-DMDBA presents a $\beta_{H R S}$ value lower than the 4-DMDBA, because the methoxy group is in orto-position, but still around two times higher than the reference compound. Similar compounds tend to change their NLO response magnitude with the position of the substituents on the phenyl ring. Such an effect was previously studied and confirmed elsewhere $[46,47]$. In those studies, the methoxy group influenced the NLO response when placed in different positions (para, ortho, and meta) of the phenyl ring, achieving the highest response when placed in the para-position of the phenyl ring. Such a tendency was also observed in the current work. Compound 4-DFDBA presented the lowest $\beta_{H R S}$ value, around three times smaller than the 4-DMDBA. Such a difference was expected, as that the methoxy group is a stronger electron donor compared to the fluor group [48], resulting in a decrease of the delocalization of the electron cloud.

The theoretical results obtained by the QCC for both the static $\left(\beta_{\mathrm{HRS}}(0 ; 0,0)\right)$ and dynamic $\left(\beta_{\text {HRS }}(-2 \omega ; \omega, \omega)\right)$ first molecular hyperpolarizability of the samples in dichloromethane medium are shown in Figure 4. From comparison with the theoretical and experimental results, a good agreement was observed for the dynamic first molecular hyperpolarizability, in particular when it is taken into account the experimental error $(20 \%)$ in HRS measurement.

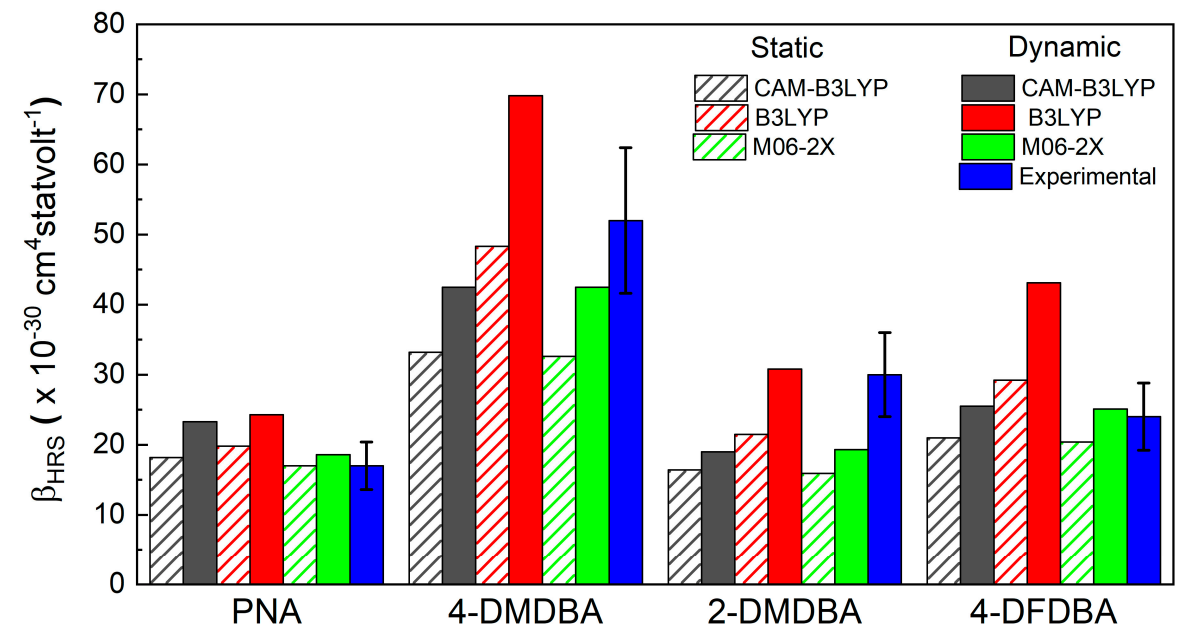

Figure 4. Experimental and theoretical values of the first molecular hyperpolarizability of all DBA derivatives studied, including the reference compound (PNA), in dichloromethane medium.

In fact, the comparison between the measured $\beta_{\mathrm{HRS}}$ values of the studied compounds with similar ones reported previously, such as chalcones derivatives [28], oxazoles dyes [29], and dibenzylideneacetones derivatives with different substituents [49], reveals the potential of using the current compounds as frequency converters. For example, the reported $\beta_{\mathrm{HRS}}$ value of a compound from the same molecular structure family (1E,4E)-1,5-bis(4-bromophenyl)penta-1,4-dien-3-one was $25 \times 10^{-30} \cdot \mathrm{cm}^{4} \cdot$ statvolt $^{-1}$ [49], which is, two times lower than the value obtained for the 4-DMDBA. Another example, an oxazole derivative 4-((5-phenyloxazol-2-yl)ethynyl)benzonitrile [29], with a longer $\pi$-electron backbone length presented a value of $45 \times 10^{-30} \cdot \mathrm{cm}^{4} \cdot$ statvolt ${ }^{-1}$, which still is lower than the 4-DMDBA.

\subsection{Two-Photon Absorption}

The broad shape of the absorption band suggests that two electronic transitions were superimposed. Spectral decomposition with Gaussian band-shape (Figure 5) revealed the absorption band consists of the two components centered at $3.36 \mathrm{eV}(369 \mathrm{~nm})$ and $3.75 \mathrm{eV}(330 \mathrm{~nm})$ for 4-DMDBA, at $3.50 \mathrm{eV}$ 
(354 nm) and $4.12 \mathrm{eV}(301 \mathrm{~nm})$ for 2-DMDBA, and at $3.67 \mathrm{eV}(338 \mathrm{~nm})$ and $4.11 \mathrm{eV}(302 \mathrm{~nm})$ for 4-DFDBA. The TPA spectra of all DBA derivatives are also presented in Figure 5, showing a $\sigma^{\text {TPA }}$ maximum value of $39 \mathrm{GM}$ at $660 \mathrm{~nm}$ for 4-DMDBA, $32 \mathrm{GM}$ at $580 \mathrm{~nm}$ for 2-DMDBA, and $20 \mathrm{GM}$ at $600 \mathrm{~nm}$ for 4-DFDBA.

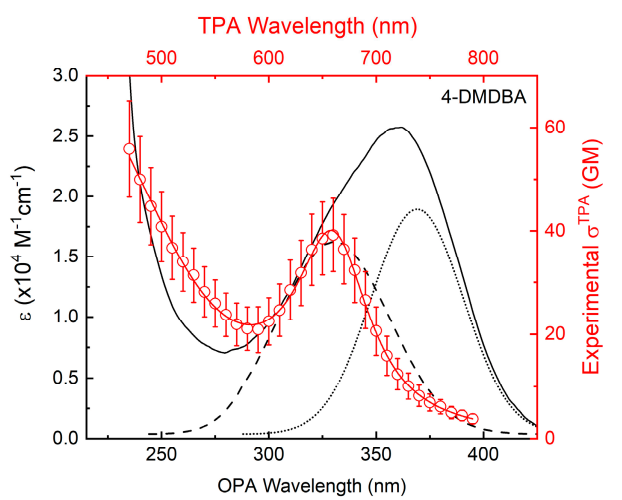

(a)

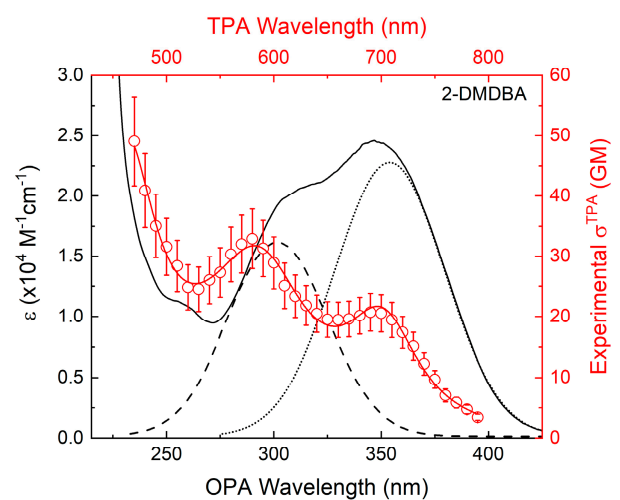

(b)

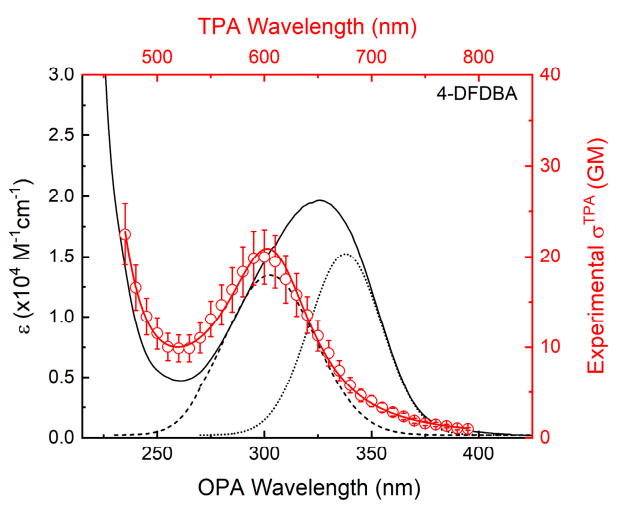

(c)

Figure 5. Experimental OPA (black solid lines) and TPA (red circles) spectra of the compounds (a) 4-DMDBA, (b) 2-DMDBA, and (c) 4-DFDBA. The dashed and dotted lines represent the Gaussian decomposition, while the solid red lines correspond to the theoretical fitting obtained with the SOS approach.

To further interpret the TPA spectra, the sum-over-states (SOS) approach was used to fit the experimental spectra (red lines in Figure 5). In this work, the samples studied presented a molecular structure close to a linear molecule and were approximately centrosymmetric (or centrosymmetric-like), making only the contribution of the three-state relevant for the TPA spectra. The following is the TPA cross-section $\left(\sigma^{T P A}\right)$ spectrum as explained by Equation (6) for one intermediate state $(k)$ and three destination states $\left(f, f^{\prime}, f^{\prime \prime}\right)[50]$ :

$$
\sigma^{T P A}(\omega)=\frac{128}{5} \frac{\pi^{3} L^{4}}{(c h n)^{2}}\left[\frac{\omega^{2}}{\left(\omega_{0 k}-\omega\right)^{2}+\Gamma_{0 k}^{2}}\left(\frac{\left|\mu_{0 k}\right|^{2}\left|\mu_{k f}\right|^{2} \Gamma_{0 f}}{\left(\omega_{0 f}-2 \omega\right)^{2}+\Gamma_{0 f}^{2}}+\frac{\left|\mu_{0 k}\right|^{2}\left|\mu_{k f^{\prime}}\right|^{2} \Gamma_{0 f^{\prime}}}{\left(\omega_{0 f^{\prime}}-2 \omega\right)^{2}+\Gamma_{0 f^{\prime}}^{2}}+\frac{\left|\mu_{0 k}\right|^{2}\left|\mu_{k f^{\prime \prime}}\right|^{2} \Gamma_{0 f^{\prime \prime}}}{\left(\omega_{0 f^{\prime \prime}}-2 \omega\right)^{2}+\Gamma_{0 f^{\prime \prime}}^{2}}\right)\right]
$$

in which $\omega$ is the pumping frequency, $c$ is the speed of light, $h$ is the Planck constant, $L$ is the Onsager local field factor, and $n$ is the refraction index (in which $n=1.424$ for dichloromethane). The spectroscopic parameters, $\omega_{i j}, \Gamma_{i j}$, and $\mu_{i j}$ correspond to transition frequency, damping constant, and transition dipole moments, respectively, of the $i \rightarrow j$ transition $\left(i=0, k\right.$ and $\left.j=k, f, f^{\prime}, f^{\prime \prime}\right)$. Most of the parameters in Equation (6) were obtained from OPA spectra, such as the transitions frequency and damping constants. In addition, the transition dipole moment $\left(\mu_{0 k}\right)$ between the ground and the $k$ excited state can be estimated from OPA spectra using [51]. 


$$
\mu_{0 k}=\sqrt{\frac{3}{8 \pi^{3}} \frac{c h}{N} \frac{1}{\omega_{0 k}} \int \alpha\left(\omega_{0 k}\right) d \omega},
$$

in which, $N$ is the number of molecules per $\mathrm{cm}^{3}$ and $\alpha$ is the absorption coefficient in $\mathrm{cm}^{-1}$. The transition dipole moments between the excited states $\left(\mu_{k f}, \mu_{k f^{\prime}}\right.$, and $\left.\mu_{k f^{\prime \prime}}\right)$ could not be obtained directly from the experiments and were determined as adjustable parameters and retrieved by the SOS approach. Table S6 in the Supplementary Material summarizes the spectroscopic parameters provided by the SOS fitting of all compounds.

Both simulated OPA and TPA spectra, using the functionals CAM-B3LYP, B3LYP, and M06-2X, are shown in Figure 6, as well as their corresponding experimental spectra. The difference of magnitude between the experimental and theoretical OPA/TPA spectra is well known and has been widely discussed elsewhere [52-54].

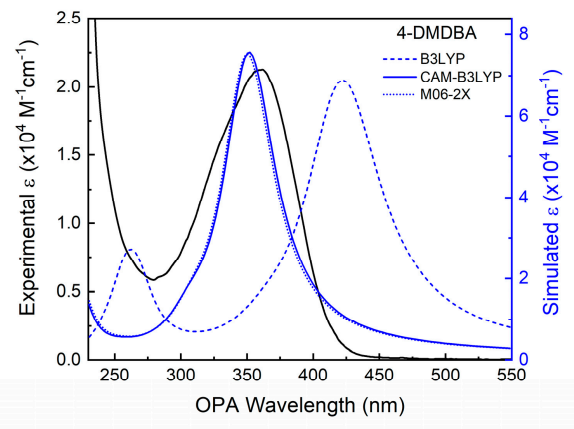

(a)

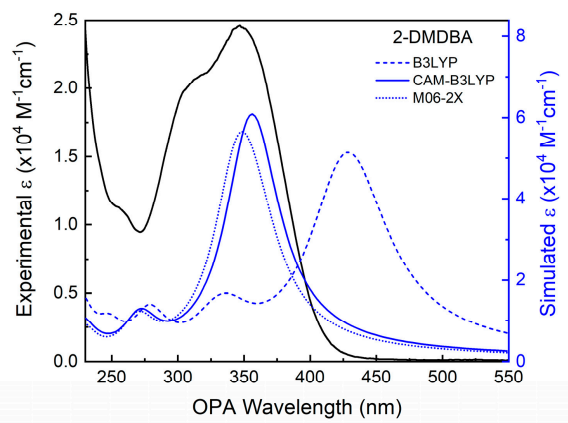

(c)

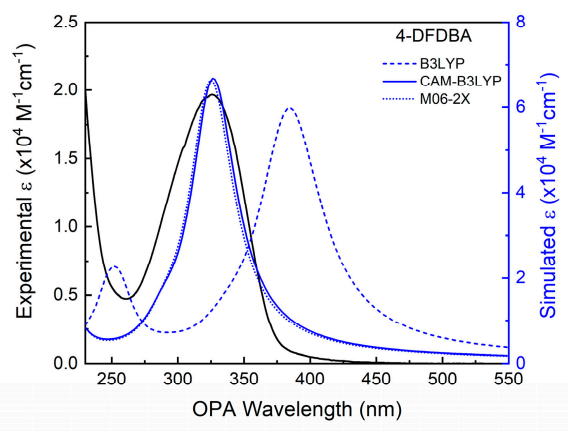

(e)

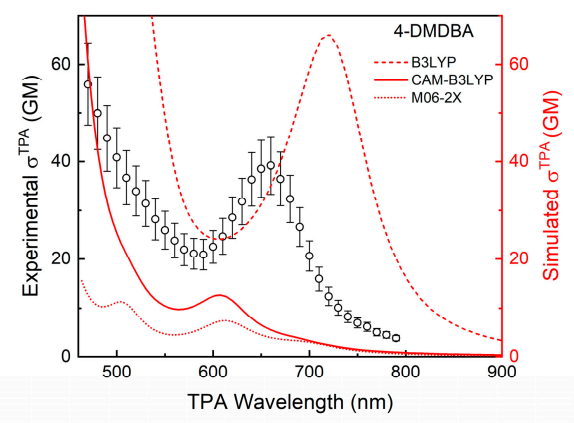

(b)

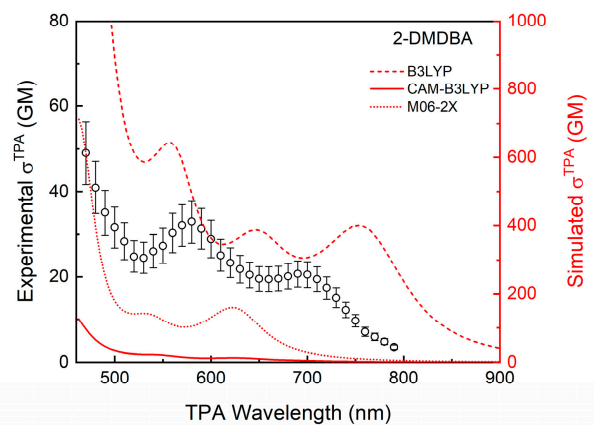

(d)

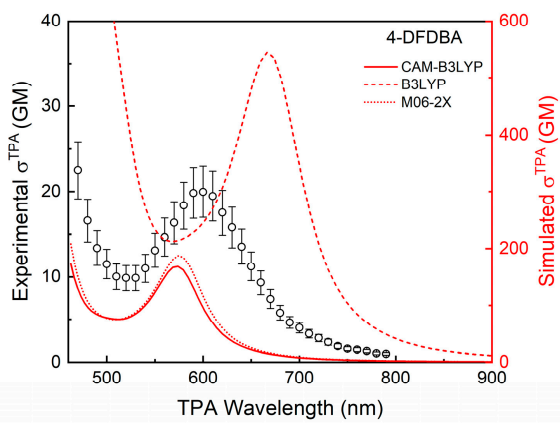

(f)

Figure 6. Experimental and simulated one-photon absorption of the DBA derivatives (a) 4-DMDBA, (c) 2-DMDBA, and (e) 4-DFDBA. Experimental and simulated two-photon absorption spectra of the DBA derivatives (b) 4-DMDBA, (d) 2-DMDBA, and (f) 4-DFDBA. Both simulated and experimental spectra were obtained in a dichloromethane medium. 
The TPA study presented here shows that all studied samples exhibit a negligible $\sigma^{T P A}$ in the IR spectral region. Based on that, no vertical excitation of any order, nor any radiative process, is expected to occur in the spectral region of interest $(1064 \mathrm{~nm})$, except for the HRS process. Consequently, no additional correction is needed to obtain a reliable first molecular hyperpolarizability. In summary, the negligible $\sigma^{T P A}$ for wavelengths longer than $800 \mathrm{~nm}$ suggests that the highest contribution for the HRS response are the electronic effects, whereas the vibrational contribution plays a small role [55]. On the other hand, it also reveals that these types of compounds are not suitable for optical limitation in the IR region. Moreover, the moderate values of $\sigma^{T P A}$ up to $40 \mathrm{GM}$ in the VIS region do not mark these compounds as potential candidates for optical limitation in the VIS region.

\section{Conclusions}

The nonlinear optical study of three dibenzylideneacetone derivatives, with a short-sized $\pi$-electron backbone length, in a dichloromethane solution, is presented in this work. The magnitude of the second-order nonlinear optical response of the studied compounds showed $\beta_{\text {HRS }}$ values of up to $52 \cdot \mathrm{cm}^{4} \cdot$ statvolt $^{-1}$, a robust value considering its short conjugation, revealing its potential to convert infrared light into visible light. The third-order nonlinear optical response, i.e., the TPA, allowed us to confirm that the $\beta_{\text {HRS }}$ values obtained have their highest contribution due to electronic effects from the HRS response. On the other hand, the negligible $\sigma^{T P A}$ values in the optical region of interest (1064 $\mathrm{nm}$ ) show that these types of compounds are not suitable for use as optical limiters in this spectral region. In summary, this work shows the potential of dibenzylideneacetone derivatives to be used as raw materials to develop optical frequency conversion photonic devices, in particular, the compound 4 -DMDBA, which presented a $\beta_{\text {HRS }}$ value three times higher than the reference compound (PNA).

Supplementary Materials: The following are available online at http://www.mdpi.com/2304-6732/7/1/8/s1, Figure S1: ${ }^{1} \mathrm{H}$ NMR Spectra of compound 2-DMDBA. Figure S2: ${ }^{13} \mathrm{C}$ NMR Spectra of compound 2-DMDBA. Figure S3: ${ }^{1} \mathrm{H}$ NMR spectra of compound 4-DMDBA. Figure S4: ${ }^{13} \mathrm{C}$ NMR spectra of compound 4-DMDBA. Figure S5: ${ }^{1} \mathrm{H}$ NMR spectra of compound 4-DFDBA. Figure S6: ${ }^{13} \mathrm{C}$ NMR spectra of compound 4-DFDBA. Figure S7: Open aperture Z-scan signature for 4-DFDBA in dichloromethane solution when excited with a femtosecond pulse tuned at $610 \mathrm{~nm}$, where the circles represent the experimental values while the solid line is the fitting obtained with Equation (3). Figure S8: Open aperture Z-scan signature for 2-DMDBA in dichloromethane solution when excited with a femtosecond pulse tuned at $580 \mathrm{~nm}$ and $690 \mathrm{~nm}$, where the symbols represent the experimental values while the solid line is the fitting obtained with Equation (3). Figure S9: Open aperture Z-scan signature for 4-DMDBA in dichloromethane solution when excited with a femtosecond pulse tuned at $660 \mathrm{~nm}$, where the squares represent the experimental values while the solid line is the fitting obtained with Equation (3). Table S1: Optimized geometries for 4-DMDBA and 2-DMDBA. Table S2: Optimized geometries for 4-DFDBA. Table S3: Excited states, transition energy, and oscillator strength for 4-DMDBA. Table S4: Excited states, transition energy, and oscillator strength for 2-DMDBA. Table S5: Excited states, transition energy, and oscillator strength for 4-DFDBA. Table S6: Spectroscopic parameters obtained through the SOS fitting of the experimental TPA spectra.

Author Contributions: Conceptualization, L.M.G.A.; methodology, L.D.B.; validation, L.D.B.; formal analysis, K.K.; investigation, F.A.S. and R.D.F.; resources, M.A.R.C.A., M.S.V. and A.M.A.; writing-original draft preparation, F.A.S.; writing-review and editing, L.M.G.A.; C.R.M.; visualization, F.A.S. and L.M.G.A.; supervision, J.J.R.J.; project administration, L.D.B.; funding acquisition, C.R.M. All authors have read and agreed to the published version of the manuscript.

Funding: This research was funded by CAPES, CNPq/MCT, FAPITEC, Nanofoton Network, INCT-FOTÔNICA, FAPESP \#2018/11283-7, FAPESP \#2016/20886-1, Army Research Laboratory W911NF-17-1-0123, Air Force Office of Scientific Research (FA9550-12-1-0028). Coordenação de Aperfeiçoamento de Pessoal de Nível Superior-Brasil (CAPES)-Finance Code 001.

Acknowledgments: The authors acknowledge the financial support from FAPESP (Fundação de Amparo à Pesquisa do Estado de São Paulo), FAPITEC (Fundação de Apoio à Pesquisa e à Inovação Tecnológica do Estado de Sergipe), and INCT de Fotônica: National Institute of Photonics.

Conflicts of Interest: The authors declare no conflict of interest. 


\section{References}

1. Mohammed, N.; Wiles, A.A.; Belsley, M.; Fernandes, S.S.; Cariello, M.; Rotello, V.M.; Raposo, M.M.M.; Cooke, G. Synthesis and characterisation of push-pull flavin dyes with efficient second harmonic generation (SHG) properties. RSC Adv. 2017, 7, 24462-24469. [CrossRef]

2. Zhang, H.; Liao, Q.; Wang, X.; Xu, Z.; Fu, H. Self-assembled organic hexagonal micro-prisms with high second harmonic generation efficiency for photonic devices. Nanoscale 2015, 7, 10186-10192. [CrossRef] [PubMed]

3. Xing, X.-S.; Sa, R.-J.; Li, P.-X.; Zhang, N.-N.; Zhou, Z.-Y.; Liu, B.-W.; Liu, J.; Wang, M.-S.; Guo, G.-C. Second-order nonlinear optical switching with a record-high contrast for a photochromic and thermochromic bistable crystal. Chem. Sci. 2017, 8, 7751-7757. [CrossRef] [PubMed]

4. Chai, Z.; Hu, X.; Wang, F.; Niu, X.; Xie, J.; Gong, Q. Ultrafast All-Optical Switching. Adv. Opt. Mater. 2017, 5, 1600665. [CrossRef]

5. Song, Y.; Chen, Y.; Jiang, X.; Liang, W.; Wang, K.; Liang, Z.; Ge, Y.; Zhang, F.; Wu, L.; Zheng, J.; et al. Nonlinear Few-Layer Antimonene-Based All-Optical Signal Processing: Ultrafast Optical Switching and High-Speed Wavelength Conversion. Adv. Opt. Mater. 2018, 6, 1701287. [CrossRef]

6. Kulyk, B.; Kerasidou, A.P.; Soumahoro, L.; Moussallem, C.; Gohier, F.; Frère, P.; Sahraoui, B. Optimization and diagnostic of nonlinear optical features of $\pi$-conjugated benzodifuran-based derivatives. RSC Adv. 2016, 6, 14439-14447. [CrossRef]

7. Rosenne, S.; Grinvald, E.; Shirman, E.; Neeman, L.; Dutta, S.; Bar-Elli, O.; Ben-Zvi, R.; Oksenberg, E.; Milko, P.; Kalchenko, V.; et al. Self-assembled organic nanocrystals with strong nonlinear optical response. Nano Lett. 2015, 15, 7232-7237. [CrossRef]

8. Hales, J.M.; Barlow, S.; Kim, H.; Mukhopadhyay, S.; Brédas, J.-L.; Perry, J.W.; Marder, S.R. Design of organic chromophores for all-optical signal processing applications. Chem. Mater. 2013, 26, 549-560. [CrossRef]

9. Chen, S.; Li, Y.; Yang, W.; Chen, N.; Liu, H.; Li, Y. Synthesis and tuning optical nonlinear properties of molecular crystals of benzothiadiazole. J. Phys. Chem. C 2010, 114, 15109-15115. [CrossRef]

10. Desiraju, G.R. Crystal engineering: From molecule to crystal. J. Am. Chem. Soc. 2013, 135, $9952-9967$. [CrossRef]

11. Ostroverkhova, O. Handbook of Organic Materials for Optical and (Opto) Electronic Devices: Properties and Applications; Elsevier: Amsterdam, The Netherlands, 2013.

12. Verbiest, T.; Houbrechts, S.; Kauranen, M.; Clays, K.; Persoons, A. Second-order nonlinear optical materials: Recent advances in chromophore design. J. Mater. Chem. 1997, 7, 2175-2189. [CrossRef]

13. Beaujean, P.; Bondu, F.; Plaquet, A.; Garcia-Amorós, J.; Cusido, J.; Raymo, F.M.; Castet, F.; Rodriguez, V.; Champagne, B. Oxazines: A new class of second-order nonlinear optical switches. J. Am. Chem. Soc. 2016, 138, 5052-5062. [CrossRef] [PubMed]

14. Miniewicz, A.; Bartkiewicz, S.; Wojaczynska, E.; Galica, T.; Zalesny, R.; Jakubas, R. Second harmonic generation in nonlinear optical crystals formed from propellane-type molecules. J. Mater. Chem. C 2019, 7, 1255-1262. [CrossRef]

15. Luo, Y.; Yuan, C.; Xu, J.; Li, Y.; Liu, H.; Semin, S.; Rasing, T.; Yang, W.; Li, Y. Controlling the growth of molecular crystal aggregates with distinct linear and nonlinear optical properties. ACS Appl. Mater. Interfaces 2017, 9, 30862-30871. [CrossRef] [PubMed]

16. Beverina, L.; Fu, J.; Leclercq, A.; Zojer, E.; Pacher, P.; Barlow, S.; Van Stryland, E.W.; Hagan, D.J.; Brédas, J.-L.; Marder, S.R.; et al. Two-photon absorption at telecommunications wavelengths in a dipolar chromophore with a pyrrole auxiliary donor and thiazole auxiliary acceptor. J. Am. Chem. Soc. 2005, 127, 7282-7283. [CrossRef]

17. Liu, X.; Wang, D.; Gao, H.; Yang, Z.; Xing, Y.; Cao, H.; He, W.; Wang, H.; Gu, J.; Hu, H. Click chemistry functionalization improving the wideband optical-limiting performance of fullerene derivatives. Phys. Chem. Chem. Phys. 2016, 18, 7341-7348. [CrossRef]

18. Bouit, P.A.; Kamada, K.; Feneyrou, P.; Berginc, G.; Toupet, L.; Maury, O.; Andraud, C. Two-photon absorption-related properties of functionalized BODIPY dyes in the infrared range up to telecommunication wavelengths. Adv. Mater. 2009, 21, 1151-1154. [CrossRef]

19. Kiran, M.S.; Anand, B.; Sai, S.S.S.; Rao, G.N. Second-and third-order nonlinear optical properties of bis-chalcone derivatives. J. Photochem. Photobiol. A Chem. 2014, 290, 38-42. [CrossRef] 
20. Shetty, T.C.S.; Raghavendra, S.; Kumar, C.C.; Dharmaprakash, S. Crystal structure and nonlinear optical absorption of a new chalcone derivative: A promising candidate for optical switching. Appl. Phys. B 2016, 122, 205. [CrossRef]

21. Reddy, N.S.K.; Badam, R.; Sattibabu, R.; Molli, M.; Muthukumar, V.S.; Sai, S.S.S.; Rao, G.N. Synthesis, characterization and nonlinear optical properties of symmetrically substituted dibenzylideneacetone derivatives. Chem. Phys. Lett. 2014, 616, 142-147. [CrossRef]

22. Rajashekar, B.; Sowmendran, P.; Sai, S.S.S.; Rao, G.N. Synthesis, characterization and two-photon absorption based broadband optical limiting in diarylideneacetone derivative. J. Photochem. Photobiol. A Chem. 2012, 238, 20-23. [CrossRef]

23. Badaeva, E.A.; Timofeeva, T.V.; Masunov, A.; Tretiak, S. Role of donor- acceptor strengths and separation on the two-photon absorption response of cytotoxic dyes: A TD-DFT Study. J. Phys. Chem. A 2005, 109, 7276-7284. [CrossRef] [PubMed]

24. Karuppasamy, P.; Sivasubramani, V.; Pandian, M.S.; Ramasamy, P. Growth and characterization of semi-organic third order nonlinear optical (NLO) potassium 3,5-dinitrobenzoate (KDNB) single crystals. RSC Adv. 2016, 6, 109105-109123. [CrossRef]

25. Thanigaimani, K.; Arshad, S.; Khalib, N.C.; Razak, I.A.; Arunagiri, C.; Subashini, A.; Sulaiman, S.F.; Hashim, N.S.; Ooi, K.L. A new chalcone structure of (e)-1-(4-bromophenyl)-3-(napthalen-2-yl) prop-2-en-1-one: Synthesis, structural characterizations, quantum chemical investigations and biological evaluations. Spectrochim. Acta Part A Mol. Biomol. Spectrosc. 2015, 149, 90-102. [CrossRef]

26. Prasad, A.A.; Muthu, K.; Meenatchi, V.; Rajasekar, M.; Agilandeshwari, R.; Meena, K.; Manonmoni, J.V.; Meenakshisundaram, S. Optical, vibrational, NBO, first-order molecular hyperpolarizability and Hirshfeld surface analysis of a nonlinear optical chalcone. Spectrochim. Acta Part A Mol. Biomol. Spectrosc. 2015, 140, 311-327. [CrossRef]

27. Franzen, P.L.; Misoguti, L.; Zilio, S.C. Hyper-Rayleigh scattering with picosecond pulse trains. Appl. Opt. 2008, 47, 1443-1446. [CrossRef]

28. Abegão, L.M.; Fonseca, R.D.; Santos, F.A.; Souza, G.B.; Barreiros, A.L.B.; Barreiros, M.L.; Alencar, M.; Mendonça, C.R.; Silva, D.L.; De Boni, L.; et al. Second-and third-order nonlinear optical properties of unsubstituted and mono-substituted chalcones. Chem. Phys. Lett. 2016, 648, 91-96. [CrossRef]

29. Abegão, L.M.; Fonseca, R.D.; Santos, F.A.; Rodrigues, J.J.; Kamada, K.; Mendonça, C.R.; Piguel, S.; De Boni, L. First molecular electronic hyperpolarizability of series of $\pi$-conjugated oxazole dyes in solution: An experimental and theoretical study. RSC Adv. 2019, 9, 26476-26482. [CrossRef]

30. Clays, K.; Persoons, A. Hyper-Rayleigh scattering in solution. Phys. Rev. Lett. 1991, 66, 2980. [CrossRef]

31. Hendrickx, E.; Clays, K.; Persoons, A. Hyper-Rayleigh scattering in isotropic solution. Acc. Chem. Res. 1998, 31, 675-683. [CrossRef]

32. Verbiest, T.; Clays, K.; Rodriguez, V. Second-Order Nonlinear Optical Characterization Techniques: An Introduction; CRC Press: Boca Raton, FL, USA, 2009.

33. Brasselet, S.; Zyss, J. Multipolar molecules and multipolar fields: Probing and controlling the tensorial nature of nonlinear molecular media. JOSA B 1998, 15, 257-288. [CrossRef]

34. Sheik-Bahae, M.; Said, A.A.; Wei, T.-H.; Hagan, D.J.; Van Stryland, E.W. Sensitive measurement of optical nonlinearities using a single beam. IEEE J. Quantum Electron. 1990, 26, 760-769. [CrossRef]

35. Gross, E.; Kohn, W. Time-dependent density-functional theory. In Advances in Quantum Chemistry; Elsevier: Amsterdam, The Netherlands, 1990; Volume 21, pp. 255-291.

36. Kohn, W.; Sham, L.J. Self-consistent equations including exchange and correlation effects. Phys. Rev. 1965, 140, A1133. [CrossRef]

37. Frisch, M.J.; Trucks, G.W.; Schlegel, H.B.; Scuseria, G.E.; Robb, M.A.; Cheeseman, J.R.; Scalmani, G.; Barone, V.; Petersson, G.A.; Nakatsuji, H.; et al. Gaussian 16 Rev. B.01; Gaussian Inc: Wallingford, CT, USA, 2016.

38. Lee, C.; Yang, W.; Parr, R.G. Development of the Colle-Salvetti correlation-energy formula into a functional of the electron density. Phys. Rev. B 1988, 37, 785. [CrossRef] [PubMed]

39. Yanai, T.; Tew, D.P.; Handy, N.C. A new hybrid exchange-correlation functional using the Coulomb-attenuating method (CAM-B3LYP). Chem. Phys. Lett. 2004, 393, 51-57. [CrossRef]

40. Zhao, Y.; Truhlar, D.G. The M06 suite of density functionals for main group thermochemistry, thermochemical kinetics, noncovalent interactions, excited states, and transition elements: Two new functionals and systematic testing of four M06-class functionals and 12 other functionals. Theor. Chem. Acc. 2008, 120, 215-241. 
41. Tomasi, J.; Mennucci, B.; Cances, E. The IEF version of the PCM solvation method: An overview of a new method addressed to study molecular solutes at the QM ab initio level. J. Mol. Struct. THEOCHEM 1999, 464, 211-226. [CrossRef]

42. Pielak, K.; Bondu, F.; Sanguinet, L.; Rodriguez, V.; Champagne, B.; Castet, F. Second-order nonlinear optical properties of multiaddressable indolinooxazolidine derivatives: Joint computational and hyper-Rayleigh scattering investigations. J. Phys. Chem. C 2017, 121, 1851-1860. [CrossRef]

43. Hirata, S.; Head-Gordon, M. Time-dependent density functional theory within the Tamm-Dancoff approximation. Chem. Phys. Lett. 1999, 314, 291-299. [CrossRef]

44. Ohta, K.; Yamada, S.; Kamada, K.; Slepkov, A.D.; Hegmann, F.A.; Tykwinski, R.R.; Shirtcliff, L.D.; Haley, M.M.; Sałek, P.; Gel'mukhanov, F.; et al. Two-photon absorption properties of two-dimensional $\pi$-conjugated chromophores: Combined experimental and theoretical study. J. Phys. Chem. A 2010, 115, 105-117. [CrossRef]

45. Woodford, J.; Pauley, M.; Wang, C. Solvent dependence of the first molecular hyperpolarizability of p-nitroaniline revisited. J. Phys. Chem. A 1997, 101, 1989-1992. [CrossRef]

46. Muhammad, S.; Al-Sehemi, A.G.; Irfan, A.; Chaudhry, A.R.; Gharni, H.; AlFaify, S.; Shkir, M.; Asiri, A.M. The impact of position and number of methoxy group (s) to tune the nonlinear optical properties of chalcone derivatives: A dual substitution strategy. J. Mol. Model. 2016, 22, 73. [CrossRef] [PubMed]

47. Lee, S.; Thomas, K.J.; Thayumanavan, S.; Bardeen, C.J. Dependence of the Two-Photon Absorption Cross Section on the Conjugation of the Phenylacetylene Linker in Dipolar Donor-Bridge-Acceptor Chromophores. J. Phys. Chem. A 2005, 109, 9767-9774. [CrossRef] [PubMed]

48. Kiran, A.J.; Rai, N.S.; Chandrasekharan, K.; Kalluraya, B.; Rotermund, F. Substituent dependence of third-order optical nonlinearity in chalcone derivatives. Jpn. J. Appl. Phys. 2008, 47, 6312. [CrossRef]

49. Santos, F.A.; Abegão, L.M.; Fonseca, R.D.; Alcântara, A.M.; Mendonça, C.R.; Valle, M.S.; Alencar, M.; Kamada, K.; De Boni, L.; Rodrigues, J., Jr. Bromo-and chloro-derivatives of dibenzylideneacetone: Experimental and theoretical study of the first molecular hyperpolarizability and two-photon absorption. J. Photochem. Photobiol. A Chem. 2019, 369, 70-76. [CrossRef]

50. Vivas, M.G.; Silva, D.L.; De Boni, L.; Bretonniere, Y.; Andraud, C.; Laibe-Darbour, F.; Mulatier, J.-C.; Zaleśny, R.; Bartkowiak, W.; Canuto, S.; et al. Experimental and theoretical study on the one-and two-photon absorption properties of novel organic molecules based on phenylacetylene and azoaromatic moieties. J. Phys. Chem. B 2012, 116, 14677-14688. [CrossRef]

51. Day, P.N.; Nguyen, K.A.; Pachter, R. TDDFT Study of One-and Two-Photon Absorption Properties: Donor$\pi$ - Acceptor Chromophores. J. Phys. Chem. B 2005, 109, 1803-1814. [CrossRef]

52. Friese, D.H.; Mikhaylov, A.; Krzeszewski, M.; Poronik, Y.M.; Rebane, A.; Ruud, K.; Gryko, D.T. Pyrrolo [3, 2-b] pyrroles-From Unprecedented Solvatofluorochromism to Two-Photon Absorption. Chem. A Eur. J. 2015, 21, 18364-18374. [CrossRef]

53. Masunov, A.; Tretiak, S. Prediction of two-photon absorption properties for organic chromophores using time-dependent density-functional theory. J. Phys. Chem. B 2004, 108, 899-907. [CrossRef]

54. Rudberg, E.; Sałek, P.; Helgaker, T.; Ågren, H. Calculations of two-photon charge-transfer excitations using Coulomb-attenuated density-functional theory. J. Chem. Phys. 2005, 123, 184108. [CrossRef]

55. Castet, F.; Rodriguez, V.; Pozzo, J.-L.; Ducasse, L.; Plaquet, A.; Champagne, B. Design and characterization of molecular nonlinear optical switches. Acc. Chem. Res. 2013, 46, 2656-2665. [CrossRef] [PubMed]

(C) 2020 by the authors. Licensee MDPI, Basel, Switzerland. This article is an open access article distributed under the terms and conditions of the Creative Commons Attribution (CC BY) license (http://creativecommons.org/licenses/by/4.0/). 